\title{
Semi-analytical Investigation of Momentum and Heat Transfer of a Non-Newtonian Fluid Flow for Specific Turbine Cooling Application Using AGM
}

\author{
H. Mirgolbabaee ${ }^{1}$ - S. T. Ledari ${ }^{2}$. \\ M. Sheikholeslami ${ }^{2}$ - D. D. Ganji ${ }^{2}$
}

Published online: 18 September 2017

(C) The Author(s) 2017. This article is an open access publication

\begin{abstract}
In this paper, a non-Newtonian fluid flow in an axisymmetric channel with porous wall for specific turbine cooling application has been considered. The purpose of this article is based on solving the nonlinear differential equations of momentum and heat transfer of the mentioned problem by utilizing a new and innovative method in semi-analytical field which is called Akbari-Ganji's method. Meanwhile, relationships between power law index, Reynolds, Prandtl and Nusselt numbers have been investigated. Results have been compared with numerical method (Runge-Kutte 4th) to achieve conclusions based on not only accuracy of the solution but also simplicity of their procedures which would have remarkable effects on the time devoted for solving process. Moreover, results are presented for various values of constant parameters and different steps of trial function due to the aim of comparison and prove that proposed solution is very accurate, simple and also have efficient convergence.
\end{abstract}

Keywords Akbari-Ganji's method (AGM) · Heat transfer · Non-Newtonian fluid flow · Nusselt number · Porous wall

\section{List of symbols}

$\begin{array}{ll}A, B & \text { Symmetric kinematic matrices } \\ C & \text { Species heat } \\ C_{n} & \text { Blade-wall temperature coefficients } \\ \frac{\delta v_{m}}{\delta x_{n}} & \text { Velocity gradients } \\ \frac{\delta a_{m}}{\delta x_{n}} & \text { Acceleration gradients }\end{array}$

H. Mirgolbabaee

h.mirgolbabaee@student.utwente.nl; hadi.mirgolbabaee@gmail.com

1 Department of Mechanical Engineering, University of Twente, P.O. Box 217, 7500 AE Enschede, The Netherlands

2 Department of Mechanical Engineering, Babol Noshirvani University of Technology, P.O. Box 484, Babol, Iran 


$\begin{array}{ll}x_{k} & \text { General coordinates } \\ f(\eta) & \text { Velocity function } \\ F & \text { Transformation of } f \\ \bar{k} & \text { Fluid thermal conductivity } \\ N & \text { Power law index in temperature distribution } \\ \mathrm{Nu} & \text { Nusselt number } \\ K_{r} & \text { Rotation parameter } \\ \mathrm{AGM} & \text { Akbari-Ganji's method } \\ p & \text { Pressure } \\ \mathrm{Pr} & \text { Prandtl number } \\ \mathrm{Re} & \text { Reynolds number } \\ T & \text { Fluid temperature } \\ q_{n}(\eta) & \text { Temperature function } \\ \tau_{i j} & \text { Stress tensor component } \\ V & \text { Injection velocity } \\ u_{r}, u_{z} & \text { Velocity components in } \mathrm{r} \text { and z directions, respectively } \\ \eta & \text { Dimensionless coordinates in z direction } \\ r, \theta, z & \text { Cylindrical coordinate symbols } \\ \phi_{k} & \text { Viscosity coefficients } \\ \varphi & \text { Dissipation function } \\ \rho & \text { Fluid density }\end{array}$

\section{Introduction}

Non-Newtonian fluid flow and heat transfer problems that are pertinent to their applications have attracted many researchers all around the world due to their technical and industrial applications such as polymer processing, electronic packing, drag reduction and cooling problems. For instance, a model of cooling turbine blades internally by continuous injection through an interior baffle was analyzed by Deburge and Han [1] in order to increase the resistance of blades against the hot steam around the blades. With respect to their considered analytical model and also their physical assumption that was based on incompressible and laminar flow, they would be able to determine the velocity and the temperature fields. Also it is noteworthy to mention that Kurtcebe and Erim [2] extended the work of Deburge and Han [1] to investigate the influence of non-Newtonian viscoelastic fluid flow on the cooling of the turbine disk. Therefore, it is obvious that non-Newtonian fluid flow could be utilized in various fields of study which some of the excellence of the previous research areas could be mentioned as [3-5]. It is also noteworthy to mention some of the recent investigations in similar area which have the same analogy and methodology and are done by several researchers around the world [6-8].

Because of the importance of mathematical formulation and analyzation in solving engineering problems, many researches from different fields devote their time to expand relevant knowledge to obtain better understanding for the mentioned case. For instance, one of the most significant feature of this ever-increasing trend could be mentioned in analytical, semianalytical and numerical technics in solving nonlinear differential equations. The main reason of widespread usage of analytical and semi-analytical methods is that by utilizing these approaches, researches would obtain a unique function in their solving procedures which could be used in other fields such as designing a control system for heat transfer 
or fluid mechanics appliances. Therefore, for the purpose of achieving the afore-mentioned fact, many researchers have tried to reach acceptable solution for differential equations due to their nonlinearity by utilizing analytical and semi-analytical methods such as: Perturbation Method [9], Homotopy Perturbation Method [10-12], Variational Iteration Method $[13,14]$, Optimal Variational Iteration Method using Adomian's Polynomials (OVIMAP) [15], Homotopy Analysis Method [14,16,17], Parameterized Perturbation Method (PPM) [18], Collocation Method (CM) [19], Adomian Decomposition Method [20,21], Variation of Parameters Method (VPM) [22] and so on.

It is worthwhile to mentioned the fact that each proposed method has their own merits which in general they could be categorized into two perspectives due to their solving procedures. We would call these two groups as: Iterate-Base and Trial Function-Base Methods. In Iterate-Base Method such as: HPM, VIM, ADM and etc., the important factor which affect the solving procedures is the number of iterations. Although in these methods we may assume a trial functions, which are based on our independent functions, however, in order to achieve solution in each step we are eligible to solve previous steps at first. According to mentioned explanations, it would be crystal clear that if the iteration results in higher steps could not be obtained by related software (such as Maple or Matlab), we would definitely face problem which will interrupt our solving procedures. Also, these methods usually take more time for obtaining solutions.

On the contrary, in Trial Function-Base Method such as: CM, LSM, Akbari-Ganji's Method (AGM) [23-30] and etc., the main factor which affect the solving procedures is considered trial function. In this methods, we assume an efficient trial function base on the problem's boundary or initial conditions which contains different constant coefficients. Afterwards, according to the basic idea of each method, we are obligated for solving the constant coefficients. In most cases the constant coefficients would be obtained easily by solving set of rudimentary polynomials. Although in these methods, number of terms in our trial function could be referred as needed iterations, nonetheless, it's essential to mention the fact that utilized constants will obtain simultaneously in solving procedures. So, in these methods the iteration problems have been eliminated.

In this article attempts have been made in order to obtain approximate solutions of the nonlinear momentum and heat transfer equations of non-Newtonian fluid flow in an axisymmetric channel with porous wall for turbine cooling applications. We have utilized a new and innovative semi-analytical method calling Akbari-Ganji's Method which was developed by Akbari and Ganji in 2014 for the first time. Since this method has been investigated by many authors to solve highly nonlinear equations in different aspects of engineering problems such as: Fluid Mechanics, Nonlinear Vibration Problems, Heat Transfer Applications, Nanofluids and etc. Some of the excellence of proposed method could be referred as [23-30].

\section{Mathematical Formulation}

\section{Flow Analysis}

Simultaneous development of flow and heat transfer for a non-Newtonian viscoelastic fluid flow on the turbine disc for cooling purposes have been considered in this study. Figure 1 represents schematic of the proposed problem in which the $r$-axis is parallel to the surface of disk and the $z$-axis is normal to it. The porous disc of the channel is $z=+L$. As it is depicted, although the wall that coincides with the $r$-axis is heated, due to the external gas 


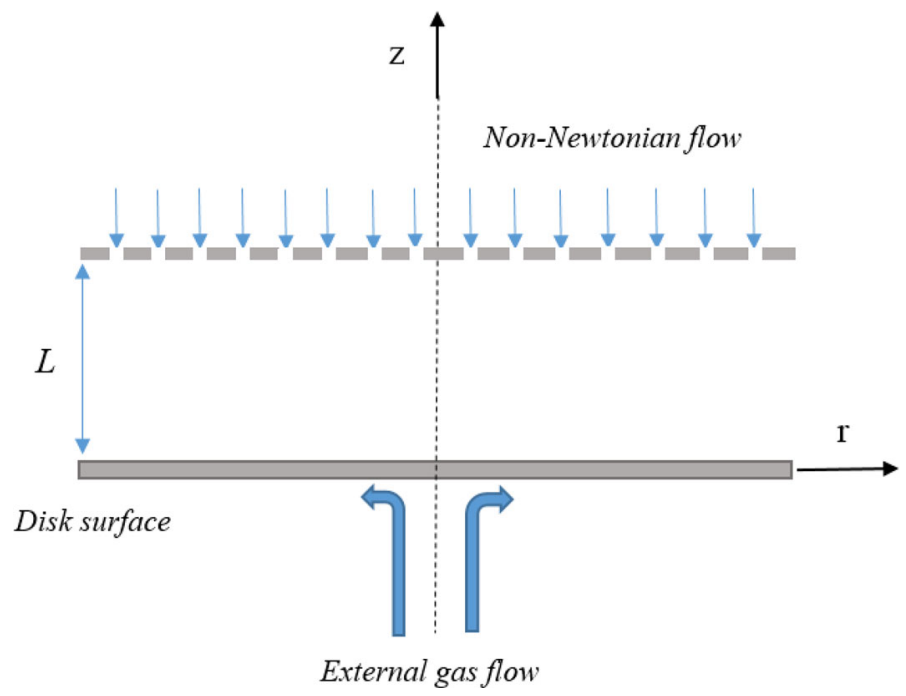

Fig. 1 Schematic diagram of the considered physical system

flow, non-Newtonian fluid is injected uniformly from the other perforated wall in order to cool the heated wall.

For a steady, axisymmetric, non-Newtonian fluid flow the following equations could be written in cylindrical coordinates. The continuity equation:

$$
\frac{\partial\left(r u_{r}\right)}{\partial r}+\frac{\partial\left(r u_{z}\right)}{\partial z}=0
$$

And the momentum equations:

$$
\begin{aligned}
& u_{r} \frac{\partial\left(u_{r}\right)}{\partial r}+u_{z} \frac{\partial\left(u_{r}\right)}{\partial z}=-\frac{1}{\rho} \frac{\partial P}{\partial r}+\frac{1}{\rho}\left[\frac{\partial \tau_{r r}}{\partial r}+\frac{1}{r}\left(\tau_{r r}-\tau_{\theta \theta}\right)+\frac{\partial \tau_{r z}}{\partial z}\right] \\
& u_{r} \frac{\partial\left(u_{z}\right)}{\partial r}+u_{z} \frac{\partial\left(u_{z}\right)}{\partial z}=-\frac{1}{\rho} \frac{\partial P}{\partial z}+\frac{1}{\rho}\left[\frac{\partial \tau_{z r}}{\partial r}+\frac{1}{r} \tau_{r z}+\frac{\partial \tau_{z z}}{\partial z}\right]
\end{aligned}
$$

Here $\rho, P$ are the density, pressure and also it is noteworthy to mention that $\tau_{r r}, \tau_{r z}, \tau_{z r}, \tau_{z z}$ are the components of stress matrix and $u_{r}, u_{z}$ are the velocity components in the $\mathrm{r}$ and $\mathrm{z}$ directions. The analytical model under consideration leads to the following boundary conditions:

$$
\begin{aligned}
& z=0 \quad \rightarrow \quad u_{r}=u_{z}=0 \\
& z=L \quad \rightarrow \quad u_{r}=0, u_{z}=-V
\end{aligned}
$$

In which $V$ is the injection velocity. For particular class of viscoelastic and viscoinelastic fluids Rivlin [31] represented that if the stress components $\tau_{i j}$ at a point $x_{k}(k=1,2,3)$ and time $t$ are assumed to be polynomials in the velocity gradient $\frac{\delta v_{m}}{\delta x_{n}}(m, n=1,2,3)$ and the acceleration gradients $\frac{\delta a_{m}}{\delta x_{n}}(m, n=1,2,3)$, and if in addition the medium is assumed to be isentropic the stress matrix can be demonstrate in the following form:

$$
\left\|\tau_{i j}\right\|=\phi_{0} I+\phi_{1} A+\phi_{2} B+\phi_{3} A^{2}+\cdots
$$


Here $I$ is the unit matrices, $A$ and $B$ are symmetric kinematic matrixes defined by:

$$
A=\left\|\frac{\delta v_{i}}{\delta x_{j}}+\frac{\delta v_{j}}{\delta x_{i}}\right\|, B=\left\|\frac{\delta a_{i}}{\delta x_{j}}+\frac{\delta a_{j}}{\delta x_{i}}+2 \frac{\delta v_{m}}{\delta x_{i}} \frac{\delta v_{m}}{\delta x_{j}}\right\|
$$

Moreover, $\phi_{k}(k=0,1,2,3)$ are polynomials in the invariants of $A, B, A^{2}$. This study is restricted to second order fluids for which $\phi_{k}(k=0,1,2,3)$ are constant and $\phi_{k}(k=4,5, .$. are zero. So that the stress components are as follows:

$$
\begin{aligned}
\tau_{r r} & =\phi_{1} A_{r r}+\phi_{2} A_{r r}^{2}+\phi_{3} B_{r r} \\
\tau_{z z} & =\phi_{1} A_{z z}+\phi_{2} A_{z z}^{2}+\phi_{3} B_{z z} \\
\tau_{\theta \theta} & =\phi_{1} A_{\theta \theta}+\phi_{2} A_{\theta \theta}^{2}+\phi_{3} B_{\theta \theta} \\
\tau_{r z} & =\phi_{1} A_{r z}+\phi_{2} A_{r z}^{2}+\phi_{3} B_{r z}
\end{aligned}
$$

In order to reach a solution of the afore-mentioned problem depicted in Fig. 1 in the case of axially symmetric flow, it would be much more convenient to define a stream function so that the continuity equation is satisfied:

$$
\psi=V r^{2} f(\eta)
$$

Where $\eta=\frac{z}{L}$ and the velocity components can be derived as:

$$
\begin{aligned}
& u_{r}=\frac{V r}{L} f^{\prime}(\eta) \\
& u_{z}=-2 V f(\eta)
\end{aligned}
$$

Using Eqs. (12)-(14) the equations of motion reduce to:

$$
\begin{aligned}
f^{\prime 2}-2 f f^{\prime \prime}= & -\frac{L^{2}}{\rho V^{2} r} \frac{\partial P}{\partial r}+\frac{\phi_{1}}{\rho V L} f^{\prime \prime \prime}+\frac{\phi_{2}}{\rho L^{2}}\left(f^{\prime \prime 2}-2 f^{\prime} f^{\prime \prime \prime}\right)+\frac{\phi_{3}}{\rho L^{2}}\left(f^{\prime \prime 2}-2 f f^{i v}\right) \\
4 f f^{\prime}= & -\frac{L^{2}}{\rho V^{2}} \frac{\partial P}{\partial z}-2 \frac{\phi_{1}}{\rho V L} f^{\prime \prime}+2 \frac{\phi_{2}}{\rho L^{2}}\left(14 f^{\prime} f^{\prime \prime}+\frac{r^{2}}{L} f^{\prime \prime} f^{\prime \prime \prime}\right) \\
& +4 \frac{\phi_{3}}{\rho L^{2}}\left(11 f^{\prime} f^{\prime \prime}+f f^{\prime \prime \prime}+\frac{r^{2}}{L} f^{\prime \prime} f^{\prime \prime \prime}\right)
\end{aligned}
$$

The pressure term can be eliminated by differentiating Eq. (15) with respect to $z$ and Eq. (16) with respect to $r$ and subtracting the resulting equations. Therefore, the following equations would be obtained:

$$
-2 f f^{\prime \prime \prime}=\frac{f^{i v}}{R e}-K_{1}\left(4 f^{\prime \prime} f^{\prime \prime \prime}+2 f^{\prime} f^{i v}\right)-K_{2}\left(4 f^{\prime \prime} f^{\prime \prime \prime}+2 f^{\prime} f^{i v}+2 f f^{v}\right)
$$

where $K_{1}=\frac{\Phi_{2}}{\rho L^{2}}, K_{2}=\frac{\Phi_{3}}{\rho L^{2}}$ is the injection Reynolds number. For $K_{2}=0$ the equation turned to:

$$
f^{i v}+2 \operatorname{Re} f f^{\prime \prime \prime}-K_{1} \operatorname{Re}\left(4 f^{\prime \prime} f^{\prime \prime \prime}+2 f^{\prime} f^{i v}\right)=0
$$

The boundary conditions are:

$$
f(0)=0, f^{\prime}(0)=0, f(1)=1, f^{\prime}(1)=0
$$




\section{Heat Transfer Analysis}

The energy equation for the present problem with viscous dissipation in non-dimensional form is given by:

$$
\begin{gathered}
\rho C\left(u_{r} \frac{\partial T}{\partial r}+u_{z} \frac{\partial T}{\partial z}\right)=\bar{k} \nabla^{2} T+\varphi \\
\varphi=\tau_{r r} \frac{\partial u_{r}}{\partial r}+\tau_{\theta \theta} \frac{u_{r}}{r}+\tau_{z z} \frac{\partial u_{z}}{\partial z}+\tau_{r z}\left(\frac{\partial u_{r}}{\partial z}+\frac{\partial u_{z}}{\partial r}\right)
\end{gathered}
$$

Here $u_{r}, u_{z}$ are the velocity components in the $r$ and $z$ directions and $V$ is the injection velocity; $\rho, P, T, C, \bar{k}$ are the density, pressure, temperature, specific heat, and heat conduction coefficient of fluid, respectively. $\varphi$ is the dissipation function.

Letting the blade wall $z=0$ temperature distribution be $T_{w}=T_{0}+\sum_{n=0}^{\infty} C_{n}\left(\frac{r}{L}\right)^{n}$ and assuming the fluid temperature to have the form of [1]:

$$
T=T_{0}+\sum_{n=0}^{\infty} C_{n}\left(\frac{r}{L}\right)^{n} q_{n}(\eta)
$$

Where $T_{0}$ is the temperature of the incoming coolant $z=L$ and neglecting dissipation effect, the following equations and boundary conditions are obtained:

$$
\begin{aligned}
& q_{n}^{\prime \prime}-\operatorname{Pr} \cdot \operatorname{Re}\left(f^{\prime} q_{n}-2 f q_{n}^{\prime}\right)=0, \quad(n=0,2,3,4, \ldots) \\
& q_{n}(0)=1, q_{n}(1)=0
\end{aligned}
$$

\section{Application of Akbari-Ganji’s Method (AGM)}

According to mentioned coupled system of nonlinear differential equations and also by considering the basic idea of the method, we rewrite Eqs. (18) and (23) in the following order:

$$
\begin{aligned}
& F(\eta)=f^{i v}+2 \operatorname{Re} f f^{\prime \prime \prime}-K_{1} \operatorname{Re}\left(4 f^{\prime \prime} f^{\prime \prime \prime}+2 f^{\prime} f^{i v}\right)=0 \\
& \Theta(\eta)=q_{n}^{\prime \prime}-\operatorname{Pr} \cdot \operatorname{Re}\left(f^{\prime} q_{n}-2 f q_{n}^{\prime}\right)=0, \quad(n=0,2,3,4, \ldots)
\end{aligned}
$$

Due to the basic idea of AGM, we have utilized a proper trial function as solution of the considered differential equation which is a finite series of polynomials with constant coefficients, as follows:

$$
\begin{aligned}
f(\eta)= & \sum_{i=0}^{9} a_{i} \eta^{i}=a_{0}+a_{1} \eta^{1}+a_{2} \eta^{2}+a_{3} \eta^{3}+a_{4} \eta^{4}+a_{5} \eta^{5} \\
& +a_{6} \eta^{6}+a_{7} \eta^{7}+a_{8} \eta^{8}+a_{9} \eta^{9} \\
\theta(\eta)= & \sum_{i=0}^{12} b_{i} \eta^{i}=b_{0}+b_{1} \eta^{1}+b_{2} \eta^{2}+b_{3} \eta^{3}+b_{4} \eta^{4}+b_{5} \eta^{5}+b_{6} \eta^{6}+b_{7} \eta^{7}+b_{8} \eta^{8} \\
& +b_{9} \eta^{9}+b_{10} \eta^{10}+b_{11} \eta^{11}+b_{12} \eta^{12}
\end{aligned}
$$




\section{Applying Boundary Conditions}

In AGM, the boundary conditions are applied in order to calculate constant coefficients of Eqs. (27) and (28) according to the following approaches:

(a) Applying the boundary conditions on Eqs. (27) and (28) are expressed as follows:

$$
u=u(B . C)
$$

Where $B C$ is the abbreviation of boundary condition. According to the above explanations, the boundary conditions are applied on Eqs. (27) and (28) in the following form:

$$
\begin{aligned}
f(0) & =0 \rightarrow a_{0}=0 \\
f(1) & =0 \rightarrow a_{9}+a_{8}+a_{7}+a_{6}+a_{5}+a_{4}+a_{3}+a_{2}+a_{1}+a_{0}=0 \\
f^{\prime}(0) & =0 \rightarrow a_{1}=0 \\
f^{\prime}(1) & =0 \rightarrow 9 a_{9}+8 a_{8}+7 a_{7}+6 a_{6}+5 a_{5}+4 a_{4}+3 a_{3}+2 a_{2}+a_{1}=0 \\
\theta(0) & =1 \rightarrow b_{0}=1 \\
\theta(1) & =0 \rightarrow \mathrm{b}_{12}+b_{11}+b_{10}+b_{9}+b_{8}+b_{7}+b_{6}+b_{5}+b_{4}+b_{3}+b_{2}+b_{1}+b_{0}=0
\end{aligned}
$$

(b) Applying the boundary conditions on the main differential equations, which in this case study are Eqs. (25) and (26), and also on theirs derivatives is done after substituting Eqs. (27) and (28) into the main differential equations as follows:

$$
\begin{aligned}
& F(f(\eta)) \rightarrow F(f(B . C))=0, \quad F^{\prime}(f(B . C))=0, \ldots \\
& \Theta(\theta(\eta)) \rightarrow \Theta(\theta(B . C))=0, \quad \Theta^{\prime}(\theta(B . C))=0, \ldots
\end{aligned}
$$

The boundary conditions are applied on the above differential equation. In fact, due to the excellence of AGM from other methods, we have to reach to set of polynomials in the processes of solution that are exactly in the same number as the overall used constant coefficients in our trial functions. Therefore, we would be able to obtain these only by simple and mundane calculations. Since in the proposed problem we have assumed two trial functions which contain 23 constant coefficients and we have 6 equations according to Eqs. (30)-(35), we have to create 17 additional equations from Eqs. (36) and (37) in order to achieve a set of polynomials which contains of 23 equations and 23 constants.

According to the above explanations we have created additional equations Eqs. (36) and (37) in the following order:

I. 6 equations have been created by calculating obtained equations from $F(0)=$ $0, \quad F(1)=0, F^{\prime}(0)=0, F^{\prime}(1)=0, F^{\prime \prime}(0)=0, F^{\prime \prime}(1)=0$

II. 11 equations have been created by calculating obtained equations from $\Theta(0)=$ $0, \Theta(1)=0, \Theta^{\prime}(0)=0, \Theta^{\prime}(1)=0, \Theta^{\prime \prime}(0)=0, \Theta^{\prime \prime}(1)=0, \Theta^{\prime \prime \prime}(0)=0, \Theta^{\prime \prime \prime}(1)=$ $0, \Theta^{\prime \prime \prime \prime}(0)=0, \Theta^{\prime \prime \prime \prime}(1)=0, \Theta^{\prime \prime \prime \prime \prime \prime}(0)=0$

The mentioned equations in (I) and (II) subsections are too large to be displayed graphically. So by utilizing the above procedures we have obtained a set of polynomials containing 23 equations and 23 constants which by solving them we would be able to obtain Eqs. (27) and (28). For instance, when $\operatorname{Re}=0.5, k=0.1, \operatorname{Pr}=2.5, n=0$, by substituting obtained 


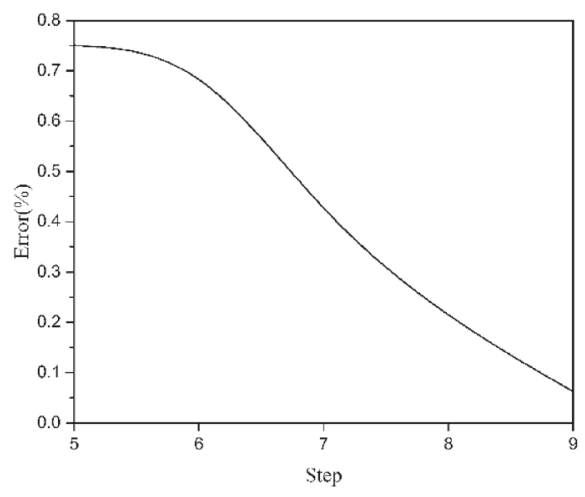

(a)

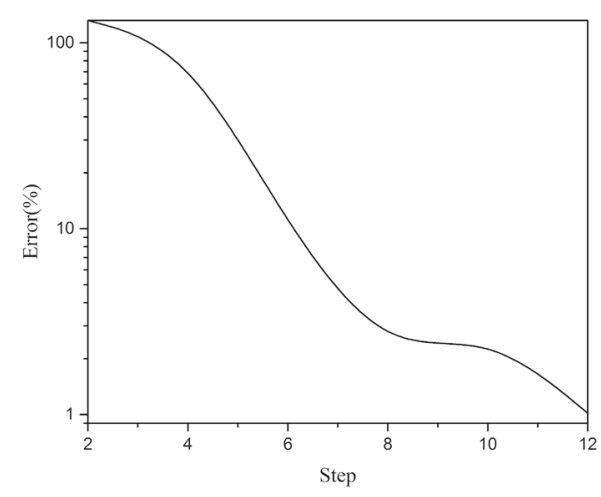

(b)

Fig. 2 a Convergence figure for $f(\eta)$ in varies terms of AGM. b Convergence figure for $q_{n}(\eta)$ in varies terms of AGM

constant coefficients from mentioned procedures Eqs. (27) and (28) could easily be yielded as follows:

$$
\begin{aligned}
f(\eta)= & -0.03009057971 \eta^{7}+0.09356884058 \eta^{6}+0.002083333333 \eta^{4}-2.227989130 \eta^{3} \\
& +3.162427536 \eta^{2} \\
\theta(\eta)= & -0.06730148307 \eta^{12}+0.4123487273 \eta^{11}-0.9074026402 \eta^{10}+0.6454071134 \eta^{9} \\
& +0.4475609223 \eta^{8}-0.6806457857 \eta^{7}+0.0002382034452 \eta^{6}-0.3821145744 \eta^{5} \\
& +.09039613610 \eta^{4}+1-1.372051844 \eta
\end{aligned}
$$

\section{Result and Discussion}

As could be detected, AGM has properly and efficiently applied through the considered problem. So in this section we have presented some of the detailed investigations through the mentioned problem using different concepts to support our work.

Figure 2 is divided into two parts which both of them represent the difference between obtained solution by AGM and numerical method (Runge-Kutte 4th) in which we have introduced error percentage as follow:

$$
\% \text { Error }=\left|\frac{u(\eta)_{N M}-u(\eta)_{A G M}}{u(\eta)_{N M}}\right| \times 100
$$

where $u(\eta)_{N M}$ is value obtained by numerical method (Runge-Kutte 4th) and $u(\eta)_{A G M}$ is value obtained by AGM. It is worthwhile to mention the fact that both Fig. 2a and b depict the convergence issue. It could be deducted that while we utilized more terms for our proposed AGM solution, the obtained error in $\eta=0.5$ would decrease.

Moreover, comparison between AGM and numerical results have been done for different values of active parameters in Fig. 3. The effects of these parameters on the velocity $(f(\eta))$ and the temperature $\left(q_{n}(\eta)\right)$ are shown. The obtained results represent that AGM has enough accuracy and efficiency so it would be applicable for solving nonlinear equations of coupled system. 


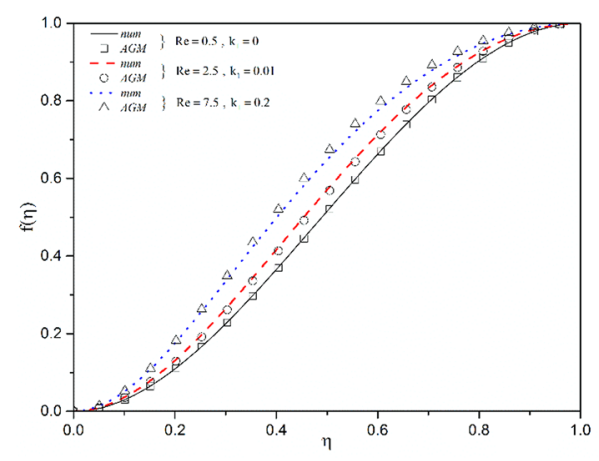

(a)

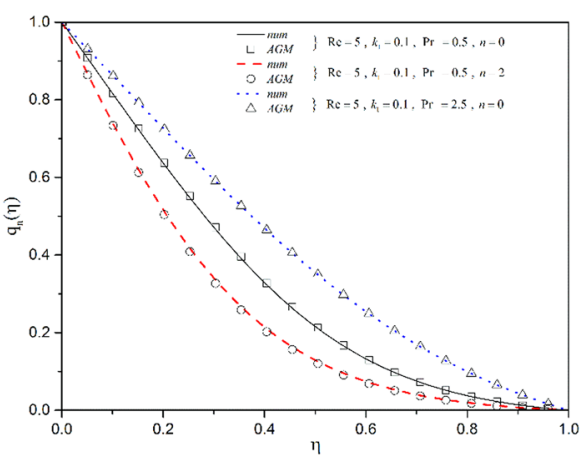

(b)

Fig. 3 a, b Comparison between numerical results and AGM solution for $f(\eta)$ and $\mathrm{q}_{n}(\eta)$

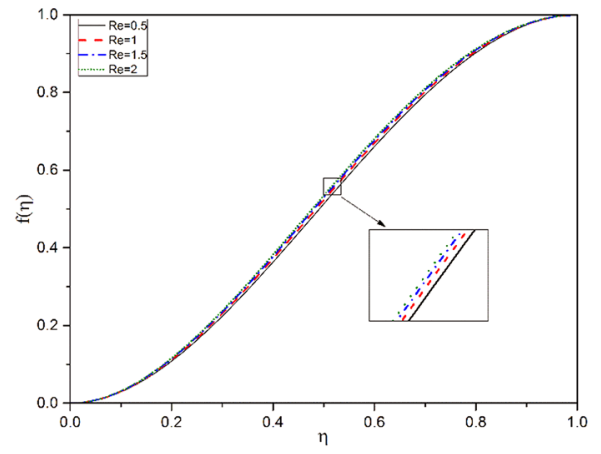

(a)

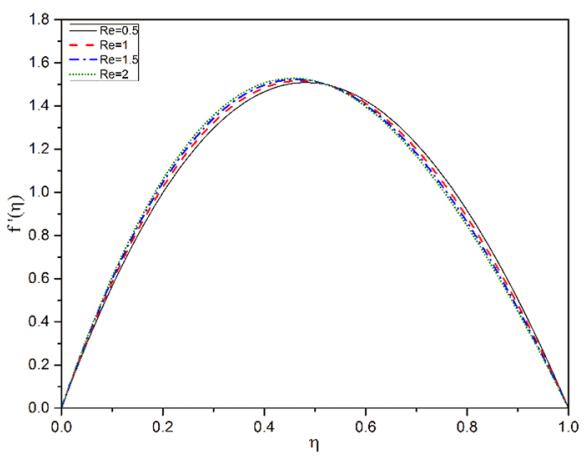

(b)

Fig. 4 Velocity component profiles $\mathbf{a} f(\eta)$ and $\mathbf{b} f^{\prime}(\eta)$ for variable $\operatorname{Re}$ at $k_{1}=0.01$

Due to the importance of the Reynolds number, which is a dimensionless number that illustrates the correlation of inertial forces to viscous forces, Fig. 4a-b and Fig. 5 have been considered. Figure $4 \mathrm{a}-\mathrm{b}$ represent the effect of Reynolds number on velocity profiles $\left(f(\eta), f^{\prime}(\eta)\right)$ where they have directly correlated. Additionally, effects of Reynolds number and injection Reynolds number on skin friction $\left(f^{\prime \prime}(0)\right)$ have been shown in Fig. 5 in which it is crystal clear that by increasing of both $\mathrm{Re}$ and $k_{1}$ parameters, the skin friction would increase substantially.

Afterwards, effect of power law index $(n)$ and the Reynolds number on temperature profile are represented. Figure 6 illustrates that by increasing the Reynolds number and power law index $(n)$, the thermal boundary layer would decrease. It is noteworthy to mention the fact that the Reynolds number changing in the value of the Reynolds number play a major role in the obtained results.

Finally, in Fig. 7 the effects of considered parameters on Nusselt number, which is equal to the dimensionless temperature gradient at the surface and provides a measure of the convection heat transfer occurring at the surface, have been discussed. Therefore, Nusselt number on the horizontal axis is plotted against Reynolds number on the vertical axis then due to Prandtl number and power law index, the various profiles are depicted. This figure demonstrates that when the Prandtl number or power law index amounts increase in the 


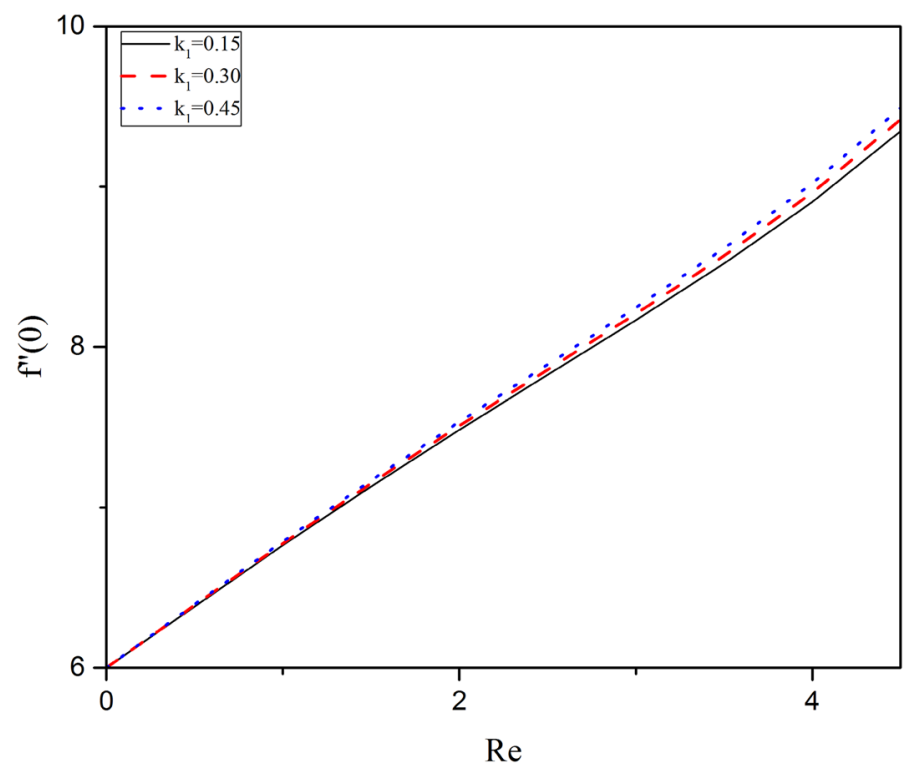

Fig. 5 Skin friction under the effect of $k_{1}$ and $\mathrm{Re}$

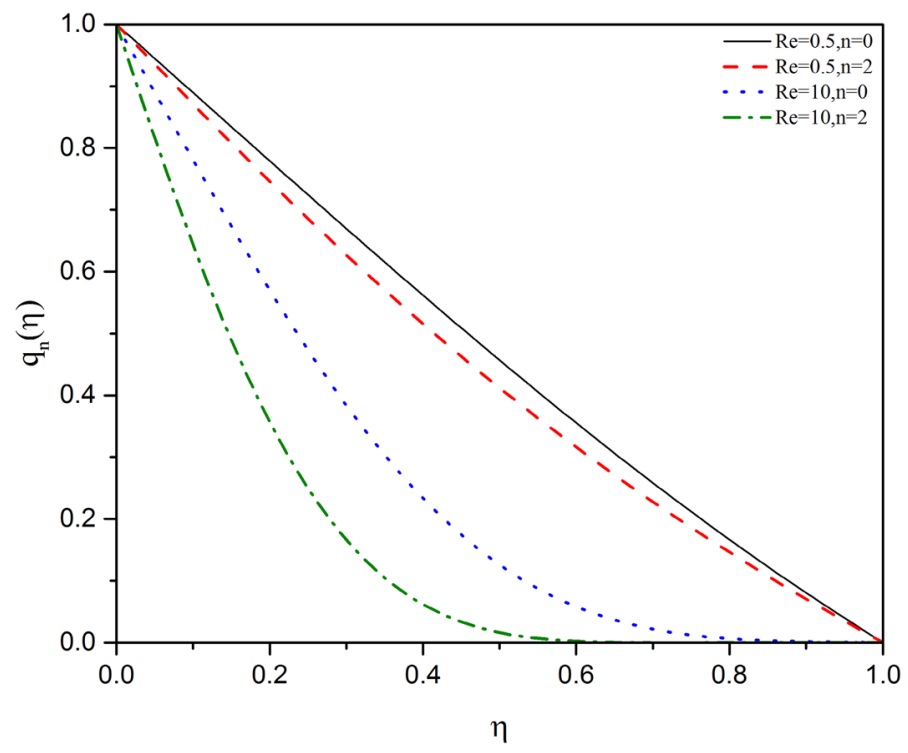

Fig. 6 Temperature profile $\left(q_{n}\right)$ at $K_{1}=0.01$

constant value of the Reynolds number, the Nusselt number would climb dramatically. Due to the fact that the Nusselt number and the thermal boundary thickness layer are negatively correlated; it is obvious that by rising the Nusselt number, with respects to its relationship with other factors, we would obtain the better cooling efficiency in industrial applications. It is also noteworthy to mention the fact that one could refer to previous articles in this field and 


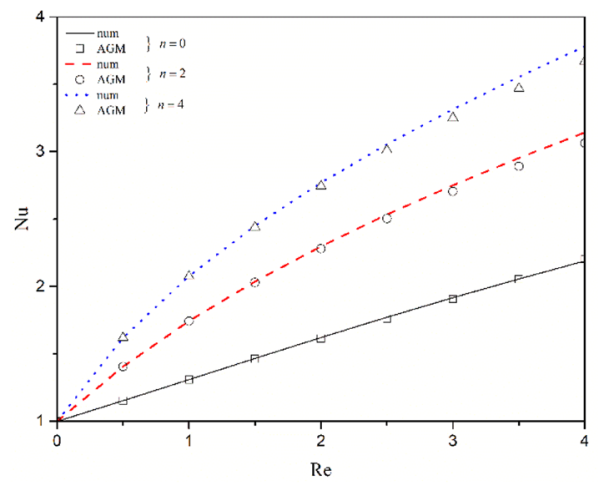

(a)

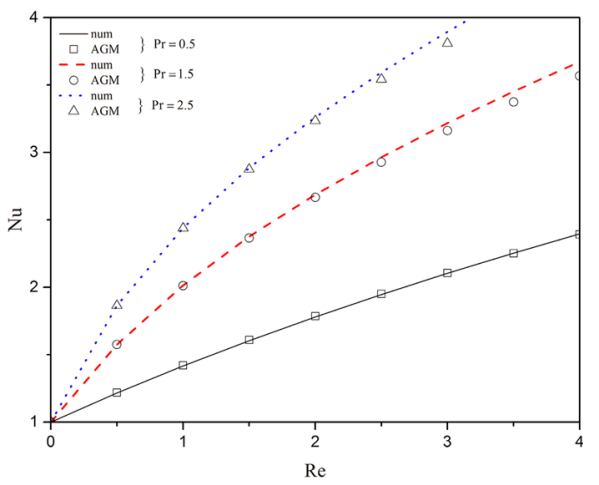

(b)

Fig. 7 Nusselt number for a variable $n$ at $K_{1}=0.01, \operatorname{Pr}=1 \mathbf{b}$ variable $\operatorname{Pr}$ at $K_{1}=0.1, n=2$

obtain even more details and results throughout other analytical and semi-analytical methods [4].

\section{Conclusion}

In this study, AGM has been utilized in order to solve nonlinear differential equations of momentum and heat transfer of a non-Newtonian fluid flow for specific turbine cooling application. Comparisons have been done among AGM and numerical method (Runge-Kutte 4th) by different parameters values. Obtained data have been revealed that AGM solutions are in a very excellence agreement with numerical solutions. Moreover, convergence figures represent that by applying more terms of AGM we would be able to obtain more accurate solutions. Therefore, it would be obvious that AGM is a convenient and qualified method for solving nonlinear problems due to its accuracy, efficiency.

Furthermore, according to achieved results, it is crystal clear that Nusselt number is an increasing function of each Reynolds number, Prandtl number and power law index. Therefore, when these properties increase during the pertinent procedures, Nusselt number would rise substantially. Finally, due to the reverse relation between Nusselt number and the thermal boundary layer thickness, we would be able to achieve more appropriate cooling characteristics in the industrial applications.

Open Access This article is distributed under the terms of the Creative Commons Attribution 4.0 International License (http://creativecommons.org/licenses/by/4.0/), which permits unrestricted use, distribution, and reproduction in any medium, provided you give appropriate credit to the original author(s) and the source, provide a link to the Creative Commons license, and indicate if changes were made.

\section{References}

1. Debruge, L.L., Han, L.S.: Heat transfer in a channel with a porous wall for turbine cooling application. ASME J. Heat Transf. 11, 385 (1972) 
2. Kurtcebe, C., Erim, M.Z.: Heat transfer of a non-Newtonian viscoinelastic fluid in an axisymmetric channel with a porous wall for turbine cooling application. Int. Commun. Heat Mass Transf. 29(7), 971-982 (2002)

3. Yuan, S.W., Finkelstein, A.B.: Heat Transfer in Laminar Pipe Flow With Uniform Coolant Injection. Trans. ASME 78, 719 (1959)

4. Ashorynejad, H.R., Javaherdeh, K., Sheikholeslami, M., Ganji, D.D.: Investigation of the heat transfer of a non-Newtonian fluid flow in an axisymmetric channel with porous wall using Parameterized Perturbation Method (PPM). J. Frank. Inst. 351(2), 701-712 (2014). doi:10.1016/j.jfranklin.2013.04.027

5. Domairry, D., Sheikholeslami, M., Ashorynejad, H.R., Gorla, R.S.R., Khani, M.: Natural convection flow of a non-Newtonian nanofluid between two vertical flat plates. Proc. Inst. Mech. Eng. Part N J. Nanomater. Nanoeng. Nanosyst. 225(3), 115-122 (2011). doi:10.1177/1740349911433468

6. Mohyud-din, S.T., Ahmed, N., Khan, U., Rashidi, M.M.: A numerical study of thermo-diffusion, diffusionthermo and chemical reaction effects on flow of a micropolar fluid in an asymmetric channel with dilating and contracting permeable walls. Eng. Comput. 34(2), 587-602 (2017). doi:10.1108/EC-03-2016-0097

7. Mohyud-Din, S.T., Ahmed, N., Khan, U., Waheed, A., Hussain, S., Darus, M.: On combined effects of heat transfer and chemical reaction for the flow through an asymmetric channel with orthogonally deformable porous walls. Math. Probl. Eng. 2016(2568785), 10 (2016). doi:10.1155/2016/2568785

8. Ahmed, N., Khan, U., Mohyud-Din, S.T.: Influence of nonlinear thermal radiation on the viscous flow through a deformable asymmetric porous channel: a numerical study. J. Mol. Liquids 225, 167-173 (2017). ISSN 0167-7322, doi:10.1016/j.molliq.2016.11.021

9. Ganji, D., Hosseini, M., Shayegh, J.: Some nonlinear heat transfer equations solved by three approximate methods. Int. Commun. Heat Mass Transf. 34, 1003-1016 (2007)

10. Sheikholeslami, M., Ganji, D.D.: Heat transfer of $\mathrm{Cu}$-water nanofluid flow between parallel plates. Powder Technol. 235, 873-879 (2013)

11. He, J.H.: Homotopy perturbation technique. Comput. Methods Appl. Mech. Eng. 178, 257-262 (1999)

12. Sfahani, M.G., Ganji, S.S., Barari, A., Mirgolbabaei, H., Domairry, G.: Analytical solutions to nonlinear conservative oscillator with fifth-order nonlinearity. Earthq. Eng. Eng. Vib. 9(3), 367-374 (2010)

13. Samaee, S.S., Yazdanpanah, O., Ganji, D.D.: New approches to identification of the Lagrange multiplier in the variational iteration method. J. Braz. Soc. Mech. Sci. Eng. 37(3), 937-944 (2015). doi:10.1007/ s40430-014-0214-3

14. Sheikholeslami, M., Ellahi, R., Ashorynejad, H.R., Domairry, G., Hayat, T.: Effects of heat transfer in flow of nanofluids over a permeable stretching wall in a porous medium. J. Comput. Theor. Nanosci. 11, 1-11 (2014)

15. Mohyud-Din, S.T., Sikander, W., Khan, U.: Optimal variational iteration method using Adomian's polynomials for physical problems on finite and semi-infinite intervals. Eur. Phys. J. Plus 132(5), 236 (2017)

16. Sheikholeslami, M., Ashorynejad, H.R., Domairry, G., Hashim, I.: Flow and heat transfer of Cu-water nanofluid between a stretching sheet and a porous surface in a rotating system. Hindawi Publ. Corp. J. Appl. Math. 2012, Article ID 421320, p 19. doi:10.1155/2012/421320

17. Umar Khan, A., Ahmed, N., Mohyud-Din, S.T.: Thermo-diffusion and diffusion-thermo effects on flow of second grade fluid between two inclined plane walls. J. Mol. Liquids 224, 1074-1082 (2016). ISSN 0167-7322, doi:10.1016/j.molliq.2016.10.068

18. Sheikholeslami, M., Rashidi, M.M., Al Saad, Dhafer M., Firouzi, F., Rokni, Houman B., Domairry, G.: Steady nanofluid flow between parallel plates considering thermophoresis and Brownian effects. J. King Saud Univ. Sci. (2015). doi:10.1016/j.jksus.2015.06.003

19. Sadeghirad, A., Kani, I.M., Rahimian, M., Astaneh, A.V.: A numerical approach based on the meshless collocation method in elastodynamics. Acta Mechanica Sinica 25(6), 857-870 (2009)

20. Asadullah, A.M., Khan, U., Ahmed, N., Mohyud-Din, S.T.: Analytical and numerical investigation of thermal radiation effects on flow of viscous incompressible fluid with stretchable convergent/divergent channels. J. Mol. Liquids 224, 768-775 (2016). ISSN 0167-7322, doi:10.1016/j.molliq.2016.10.073

21. Adnan, N.A., Khan, U., Mohyud-Din, S.T.: Influence of thermal radiation and viscous dissipation on squeezed flow of water between Riga plates saturated with carbon nanotubes. Colloids Surf. A Physicochem. Eng. Asp. 522, 389-398 (2017). ISSN 0927-7757, doi:10.1016/j.colsurfa.2017.02.083

22. Sikander, W., Khan, U., Ahmed, N., Mohyud-Din, S.T.: Optimal solutions for homogeneous and nonhomogeneous equations arising in physics. Results Phys. 7, 216-224 (2017). ISSN 2211-3797, doi:10. 1016/j.rinp.2016.12.018

23. Akbari, M.R., Ganji, D.D., Majidian, A., Ahmadi, A.R.: Solving nonlinear differential equations of Vanderpol, Rayleigh and Duffing by AGM. Front. Mech. Eng. 9(2), 177-190 (2014)

24. Sheikholeslami, M., Jalili, P., Ganji, D.D.: Magnetic field effect on nanofluid flow between two circular cylinders using AGM, Alexandria Eng. J. (2017). ISSN 1110-0168, doi:10.1016/j.aej.2017.02.010 
25. Ledari, S.T., Mirgolbabaee, H., Ganji, D.D.: Heat transfer analysis of a fin with temperature dependent thermal conductivity and heat transfer coefficient. New Trends Math. Sci. 2, 55-69 (2015)

26. Mirgolbabaee, H., Ledari, S.T., Ganji, D.D.: New approach method for solving Duffing-type nonlinear oscillator. Alexandria Eng. J. 55(2), 1695-1702 (2016). ISSN 1110-0168, doi:10.1016/j.aej.2016.03.007

27. Mirgolbabaee, H., Ledari, S.T., Ganji, D.D.: An assessment of a semi analytical AG method for solving nonlinear oscillators. New Trends Math. Sci. 1, 283-299 (2016). doi:10.1016/j.aej.2016.03.007

28. Ledari, S.T., Mirgolbabaee, H., Ganji, D.D.: An assessment of a semi analytical AG method for solving two-dimension nonlinear viscous flow. Int. J. Nonlinear Anal. Appl. 6(2), 47-64 (2015). doi:10.22075/ IJNAA.2015.270

29. Mirgolbabaee, H., Ledari, S.T., Zadeh, N.M., Ganji, D.D.: Investigation of the nonlinear equation of the circular sector oscillator by Akbari-Ganji's method. J. Taibah Univ. Sci. (2017). ISSN 1658-3655, doi:10. 1016/j.jtusci.2016.11.001

30. Mirgolbabaee, H., Ledari, S.T., Ganji, D.D.: Semi-analytical investigation on micropolar fluid flow and heat transfer in a permeable channel using AGM. J. Assoc. Arab Univ. Basic Appl. Sci. (2017). ISSN 1815-3852, doi:10.1016/j.jaubas.2017.01.002

31. Rivlin, R.S.: Stress-Deformation Relations for Isotropic Materials. J. Ration. Mech. Anal. 4, 323 (1955) 